\title{
A Randomized Clinical Study on Acupuncture Therapy for Relieving Sciatica Caused by Lumbar Disc Herniation
}

\author{
CHENG LI, TIANQIANG LI ${ }^{1}$, XINJIAN MA², CHENFEI NI ${ }^{3}, X^{2}$ AOLI WEI ${ }^{4}$ AND SHINIAN ZHANG ${ }^{4 *}$
}

Department of Accupuncture, Wuxi Integrated Chinese and Western Medicine Hospital, Affiliated Hospital of Nanjing University of Chinese Medicine, 'Department of Orthopaedics, Feixian People's Hospital, '2Department of Chinese Medicine, Wuxi Guangrui and Tongjiang Community Health Service Center, ${ }^{3}$ Department of Accupuncture, the Affiliated Hospital of Jiangnan University, ${ }^{4}$ Department of Massage, Jiangsu Provincial Hospital of Chinese Medicine, Affiliated Hospital of Nanjing University of Chinese Medicine, No. 155 Hanzhong Road, Nanjing, Jiangsu 210029, China

\section{Li et al.: Clinical Study on Acupuncture Therapy}

Acupuncture has been proved to be effective in many diseases, but the effect of acupuncture on sciatica caused by lumbar disc herniation is not clear. Our aim was to explore the efficacy and safety of acupuncture on sciatica caused by lumbar disc herniation. A total of 73 lumbar disc herniation patients with sciatica in our hospital were selected and randomly divided into the study group (acupuncture, $n=37$ ) and control group (sham acupuncture, $n=36$ ). The results showed that there were no significant differences in leg pain visual analogue scale, low back pain visual analogue scale or Oswestry Disability Index score at baseline between the study group and the control group. Compared with baseline, patients in both groups improved significantly in leg pain visual analogue scale, low back pain visual analogue scale and Oswestry Disability Index score. During treatment, improvements in leg pain visual analogue scale, low back pain visual analogue scale and Oswestry Disability Index score were all faster for patients in the study group. However, this short term benefit of acupuncture therapy was no longer significant by $16^{\text {th }} \mathbf{w}$. In addition, acupuncture therapy achieved a faster rate of perceived recovery. In terms of safety, acupuncture has been proved to be safe without obvious side effects. In acupuncture can effectively relieve sciatica caused by lumbar disc herniation in the early stage and can be used as an important auxiliary means of early treatment.

Key words: Lumbar disc herniation, sciatica, acupuncture, malnutrition

Sciatica, as one of the most common complications of lumbar disc process, has a high clinical incidence ${ }^{[1,2]}$. Epidemiological survey shows that the global prevalence of sciatica is between $1.6 \%$ and $4.3 \% 0^{[3]}$. The root sciatica caused by lumbar disc herniation (LDH) accounts for more than $90 \%$ of the population of sciatic nerve ${ }^{[4]}$. At present, the treatment of the disease is mainly divided into surgical treatment, epidural injection and conservative treatment. However, there are some disadvantages in these treatment methods, so it is necessary to find a safe, low side effects and low cost auxiliary treatment method ${ }^{[5-7]}$. In the traditional Chinese treatment, acupuncture can effectively relieve the pain, numbness and other symptoms caused by sciatica, improve physical dysfunction, so many people seek acupuncture treatment ${ }^{[8]}$. Previous studies have shown that acupuncture has certain efficacy in alleviating pain and dysfunction caused by the disease ${ }^{[9]}$. However, the systematic evaluation conclusion of the randomized controlled trial is that the efficacy of acupuncture in the treatment of sciatica is not accurate and there is no sufficient evidence to prove that the efficacy of acupuncture is better than other recognized therapies ${ }^{[10]}$. Moreover, the long term treatment of acupuncture is not sure and its level of evidence in foreign clinical guidelines is very low. Therefore, it is of practical significance to design high quality clinical randomized controlled trials to compare the efficacy of acupuncture and other therapies, as well as to evaluate the shortterm and long-term efficacy of acupuncture in the treatment of root sciatica ${ }^{[11,12]}$. In our study, we randomly divided the selected patients into acupuncture group and control group and systematically evaluated acupuncture on lumbago and leg pain, short-term effect and long-term effect. These results will provide some

*Address for correspondence

E-mail: zhangshinian@sina.com 
guidance for acupuncture treatment of sciatica. A total of 73 lumbar disc herniation patients with sciatica in Jiangsu Provincial Hospital of Chinese Medicine from August 2017 to July 2019 were selected and randomly divided into the study group (acupuncture, $n=37$ ) and control group (sham acupuncture, $n=36$ ). The other treatments of the two groups were the same. Inclusion criteria-Patients with unilateral sciatica who meet the diagnostic criteria; the course of disease is $<12 \mathrm{w}$; Age between 18 and $80 \mathrm{y}$; Volunteer to participate in the trial and sign the informed consent. Those who meet the above four items can be included in this study. Exclusion criteria-Patients requiring absolute surgical treatment: such as foot ptosis, cauda equina syndrome or severe lumbar disc herniation; After 3 mo of strict conservative treatment, the symptoms continued to worsen; Patients who have complicated with adhesion of nerve root, obvious atrophy of muscle, or positive straight leg elevation test of healthy leg; Patients with severe autoimmune diseases, heart, liver, kidney, hematopoietic system diseases or general malnutrition; Patients with cognitive impairment; Patients who have been treated with this protocol in the past $30 \mathrm{~d}$. Where any one of the above is included. Study group-The patients took the prone position and used $75 \%$ alcohol cotton ball to sterilize the operation site and then fixed the adhesive pad (in order to ensure the blind method, the acupuncture group placed the same fixed adhesive pad as the sham acupuncture group). Use acupuncture needle to directly prick "Dachangshu" $40 \mathrm{~mm}-70 \mathrm{~mm}$, so that the patient will have a local feeling of acid swelling and it is better to radiate electric shock to the lower limbs once; lift the needle up to $2 \mathrm{~mm}$ after getting Qi; after getting gas from local acid swelling, carry out small scale and uniform lifting, insertion and twisting for three times. Keep the needle for $30 \mathrm{~min}$. During the period, the needle should be done once every $10 \mathrm{~min}$ and 3 times in total ("large intestine Shu" cannot be done). Hold the sterilized cotton ball with the thumb index finger in one hand and gently press it on the acupuncture part, hold the needle in the other hand and slowly pull out the needle and press the pinhole for a while after the needle is pulled out to prevent bleeding. Control group-The acupoint selection and manipulation of the sham acupuncture group were the same as that of the acupuncture group, but the needle did not penetrate into the skin, only contacted the skin surface and the other matters were the same as that of the acupuncture group. In principle, after the last treatment every week, the patients self-rated the average value of visual analogue scale (VAS) as of lumbago and leg pain $24 \mathrm{~h}$ before the treatment. The evaluator assisted the patient to mark a point on a simulated VAS with a length of $100 \mathrm{~mm}$, which can best represent the intensity of their own pain. The scale starts from $0 \mathrm{~mm}$, ends at $100 \mathrm{~mm}, 0 \mathrm{~mm}$ represents no pain, $100 \mathrm{~mm}$ represents the most pain and the middle part represents different degrees of pain. The distance between the point marked by the patient and $0 \mathrm{~mm}$ was used as the score of pain intensity. Oswestry Disability Index (ODI) is composed of 10 problems, such as pain intensity, self care level, lifting, walking, sitting, standing, sleeping, sexual life, social life and tourism. Each question includes 6 options, the first option is 0 and the later one is 1, 2, 3, 4 and 5. The highest score of each question is 5. Scoring method: actual score/highest possible score $\times 100 \%$. For example, if all 10 questions are answered, 0di score $=$ actual score $/ 50 \times 100 \%$; if one question is not answered, the highest possible score minus the highest score of the unfilled item, that is, 0di score=actual score $/ 45 \times 100 \%$. The higher the scale score, the more serious the patient's condition. This was dichotomized into satisfactory recovery (completely and much recovered) and unsatisfactory recovery (slightly recovered, no change, slightly worse, much worse and worse than ever). With implementation methods, we refer to previous research ${ }^{[13]}$. The data in this study were analyzed using Statistical package for the social sciences (SPSS) 17.0 statistical software. The proportional data were represented as (n, \%) and were checked by chi square. Measurement data were expressed in the form of $(\mathrm{x} \pm \mathrm{s})$ and were analyzed by student $T$ test. $\mathrm{p}<0.05$ meant the difference was significant. There were no significant differences in gender, age or BMI values between the study group and the control group ( $>>0.05)$. Besides, no difference was found in the smoking and drinking population between the two groups $(\mathrm{p}>0.05)$, as shown in Table 1 . The longest course of disease was $100 \mathrm{w}$ and the shortest was $16 \mathrm{w}$ in the study group, while the longest was 134 $\mathrm{w}$ and the shortest was $14 \mathrm{w}$ in the control group. In addition, no difference was found in the location of LDH between the two groups ( $p>0.05)$. Besides, there were no significant differences in leg pain VAS, low back pain VAS or ODI score at baseline between the study group and the control group ( $>0.05)$, as shown in Table 2. Compared with baseline, patients in both groups improved significantly in leg pain VAS, low back pain VAS and ODI score $(\mathrm{p}<0.05)$. Interestingly, during treatment, improvements in leg pain VAS, low back pain VAS and ODI score were all faster for patients in the study group. However, this short-term benefit of 
acupuncture therapy was no longer significant by $16^{\text {th }}$ $\mathrm{w}$, as shown in Table 3. The 7-point Likert scale of global perceived recovery was used to evaluate the global perceived effect. At $4^{\text {th }} \mathrm{w}$, acupuncture therapy achieved a faster rate of perceived recovery. By the end of follow-up $\left(16^{\text {th }} \mathrm{w}\right)$, however, patients in both two groups had experienced satisfactory recovery without a significant difference, as shown in Table 4. In the study group, there were 2 cases $(5.4 \%)$ with pinhole hemorrhage (mild) after acupuncture and 1 case $(2.7 \%)$ with acupuncture pain. No adverse events ( $0 \%)$ happened in the control group. There was no significant difference in the rate of adverse events between the two groups, as shown in Table 5. Sciatica is not a specific disease, but as a symptom in a variety of diseases, LDH is the main cause of sciatica ${ }^{[14]}$. There are many kinds of conservative treatment, such as traction and massage, which are commonly used in domestic clinical practice. However, many elderly patients with severe osteoporosis are not suitable for traction and massage. And these therapies are short-term effective, long-term effect is not accurate. At present, radicular sciatica is still the most effective way to treat the root sciatic nerve than surgery, but surgery has the risk of causing spinal instability, extensive epidural fibrosis, many elderly patients with more complications are not suitable for surgery and the cost of surgery is higher ${ }^{[15-17]}$. Previous studies have showed that the mechanism of acupuncture analgesia involves the whole nervous system, including the central nervous system and its nucleus, peripheral nerve and its conduction system and is closely related to neurohumoral and mediators ${ }^{[9]}$. Neurotransmitters play an important role in the process of acupuncture analgesia, among which opioid peptide, 5-Light tryptamine, norepinephrine, acetylcholine and other neurotransmitters play a promoting role in acupuncture analgesia and anti opioids can block the analgesic effect of acupuncture; at the same time, acupuncture can also reduce inflammatory reaction, so as to play an analgesic role ${ }^{[14,15]}$. In our study, we used false acupuncture as a control method and special non piercing blunt head needle set. This kind of needle set has no obvious difference from the common needle set in appearance and it is simple to make, low in cost, easy to gain the trust of patients. The clinical cross test shows that the blunt needle has a good blind effect and can be used as a sham acupuncture control. The main symptom of sciatica is lower extremity radiation pain. According to the related research at home and abroad, the degree of leg pain is the main index to evaluate the severity of sciatica. Low back pain is another common symptom of chronic sciatica caused by lumbar disc herniation, so the degree of low back pain (visual analogue scale) is included in one of the secondary outcome indicators of the efficacy of this trial ${ }^{[18]}$. In this study, ODI questionnaire was used to evaluate the dysfunction. In general, if the ODI score is less than $34 \%$, it is considered that there is serious dysfunction ${ }^{[19]}$. Our results demonstrated that compared with baseline, patients in both groups improved significantly in leg pain VAS, low back pain VAS and ODI score. Interestingly, during treatment, improvements in leg pain visual analogue scale, low back pain VAS and ODI score were all faster for patients in the study group. However, this short-term benefit of acupuncture therapy was no longer significant by $16^{\text {th }} \mathrm{w}$. In addition, at $\mathrm{w} 4$, acupuncture therapy achieved a faster rate of perceived recovery. In conclusion, acupuncture can effectively relieve sciatica caused by lumbar disc herniation in the early stage and can be used as an important auxiliary means of early treatment. However, the long-term effect is still uncertain, which needs to be further confirmed by increasing the sample size.

TABLE 1: COMPARISON OF GENERAL INFORMATION BETWEEN THE TWO GROUPS

\begin{tabular}{|c|c|c|c|}
\hline & Study group $(n=37)$ & Control group $(n=36)$ & $\mathrm{p}$ \\
\hline Gender & & & 0.740 \\
\hline Male (\%) & 11 (29.7\%) & 12 (33.3 \%) & \\
\hline Female (\%) & $26(70.3 \%)$ & $24(66.7 \%)$ & \\
\hline Age $(y)$ & $56.91 \pm 8.33$ & $57.28 \pm 9.74$ & 0.697 \\
\hline BMI $\left(\mathrm{kg} / \mathrm{m}^{2}\right)$ & $25.79 \pm 3.05$ & $26.01 \pm 3.99$ & 0.234 \\
\hline Smoking & & & 0.718 \\
\hline Yes & $6(16.2 \%)$ & 7 (19.4\%) & \\
\hline No & $31(83.8 \%)$ & $29(80.6 \%)$ & \\
\hline Drinking & & & 0.818 \\
\hline Yes & $8(21.6 \%)$ & 7 (19.4\%) & \\
\hline No & $29(78.4 \%)$ & $29(80.6 \%)$ & \\
\hline
\end{tabular}


TABLE 2: COMPARISON OF DISEASE RELATED INDEXES AT BASELINE BETWEEN THE TWO GROUPS

\begin{tabular}{|c|c|c|c|}
\hline & Study group $(n=37)$ & Control group $(n=36)$ & $\mathrm{p}$ \\
\hline Course of disease (w) & $34(26)$ & $30(24)$ & 0.845 \\
\hline \multicolumn{4}{|l|}{ Location of LDH } \\
\hline $\mathrm{L}_{3}^{-}$ & & & 0.278 \\
\hline Yes & 18 & 13 & \\
\hline No & 19 & 23 & \\
\hline $\mathrm{L}_{4}^{-}{ }_{5}$ & & & 0.944 \\
\hline Yes & 27 & 26 & \\
\hline No & 10 & 10 & \\
\hline $\mathrm{L}_{5}-\mathrm{S}_{1}$ & & & 0.118 \\
\hline Yes & 25 & 30 & \\
\hline No & 12 & 6 & \\
\hline Leg pain visual analogue scale & $61.35 \pm 15.23$ & $60.87 \pm 16.01$ & 0.682 \\
\hline $\begin{array}{l}\text { Low back pain visual analogue } \\
\text { scale }\end{array}$ & $56.34 \pm 21.34$ & $57.10 \pm 20.99$ & 0.972 \\
\hline $\begin{array}{l}\text { Oswestry Disability Index } \\
\text { score }\end{array}$ & $44.22 \pm 15.82$ & $43.78 \pm 17.92$ & 0.735 \\
\hline
\end{tabular}

TABLE 3: COMPARISON OF THE LEG PAIN AND LOW BACK PAIN VAS AND ODI SCORE BETWEEN THE TWO GROUPS

\begin{tabular}{|c|c|c|c|}
\hline & Study group $(n=37)$ & Control group $(n=36)$ & $\mathrm{p}$ \\
\hline \multicolumn{4}{|c|}{ Leg pain VAS } \\
\hline $1^{\text {st }} W$ & $48.39 \pm 12.35^{*}$ & $51.56 \pm 13.24^{*}$ & 0.233 \\
\hline $2^{\text {nd }} w$ & $41.79 \pm 9.66^{*}$ & $48.28 \pm 10.12^{*}$ & 0.057 \\
\hline $3^{\text {rd }} \mathrm{W}$ & $36.67 \pm 7.47^{*}$ & $43.23 \pm 9.01^{*}$ & 0.041 \\
\hline $4^{\text {th }} w$ & $30.12 \pm 4.23^{*}$ & $40.89 \pm 6.22^{*}$ & 0.023 \\
\hline $16^{\text {th }} w$ & $29.45 \pm 5.23^{*}$ & $30.34 \pm 7.82^{*}$ & 0.434 \\
\hline \multicolumn{4}{|c|}{ Low back pain VAS } \\
\hline $1^{\text {st }} W$ & $44.18 \pm 14.12^{*}$ & $49.78 \pm 15.82^{*}$ & 0.324 \\
\hline $2^{\text {nd }} w$ & $34.23 \pm 12.23^{*}$ & $43.73 \pm 14.07^{*}$ & 0.044 \\
\hline $3^{\text {rd }} w$ & $31.28 \pm 9.87^{*}$ & $40.32 \pm 10.76^{*}$ & 0.039 \\
\hline $4^{\text {th }} w$ & $27.33 \pm 7.32 *$ & $37.19 \pm 8.99^{*}$ & 0.022 \\
\hline $16^{\text {th }} w$ & $25.68 \pm 8.22^{*}$ & $27.11 \pm 9.82^{*}$ & 0.730 \\
\hline \multicolumn{4}{|c|}{ ODI score } \\
\hline $4^{\text {th }} \mathrm{W}$ & $30.16 \pm 9.23^{*}$ & $35.72 \pm 10.02^{*}$ & 0.046 \\
\hline $16^{\text {th }} \mathrm{W}$ & $28.64 \pm 9.78^{*}$ & $29.23 \pm 8.99^{*}$ & 0.642 \\
\hline
\end{tabular}

Note: Compared with baseline, ${ }^{*} \mathrm{p}<0.05$

TABLE 4: COMPARISON OF PATIENTS' SATISFACTORY RECOVERY BETWEEN THE TWO GROUPS

\begin{tabular}{lccc}
\hline & Study group $(\mathrm{n}=37)$ & Control group $(\mathrm{n}=36)$ & $\mathrm{p}$ \\
\hline $4^{\text {th }} \mathrm{W}$ & 26 & 10 & 0.000 \\
Satisfactory recovery & 11 & 26 & 0.530 \\
Unsatisfactory recovery & & & \\
$16^{\text {th }} \mathrm{W}$ & 30 & 27 & 9 \\
Satisfactory recovery & 7 & & \\
Unsatisfactory recovery & & & \\
\hline
\end{tabular}

TABLE 5: COMPARISON OF SAFETY BETWEEN THE TWO GROUPS

\begin{tabular}{lccc}
\hline & Study group $(\mathrm{n}=37)$ & Control group $(\mathrm{n}=36)$ & $\mathrm{P}$ \\
\hline Bleeding & 2 & 0 & 0.1572 \\
Yes & 35 & 36 & 0.321 \\
No & & & 0 \\
Other adverse events & 1 & 36 & \\
Yes & 36 & 36 & \\
No & 36 & & \\
\hline
\end{tabular}




\section{Declaration of interest:}

The authors report no conflicts of interest.

\section{REFERENCES}

1. Wang Y, Dai G, Li L, Liu L, Jiang L, Li S, et al. Transcriptome signatures reveal candidate key genes in the whole blood of patients with lumbar disc prolapse. Exp Ther Med 2019;18(6):4591-602.

2. Bouma GJ, van den Brink W, Miller LE, Wolfs JF, Arts MP. Does Patient Blinding Influence Clinical Outcomes After Annular Closure Device Implantation? A Propensity ScoreMatched Analysis. Orthop Res Rev 2019;11:177-82.

3. Asahar SF, Abd Malek K, Zohdi WN, Peter AB. Chronic back pain in a young female patient: A case of ependymoma originating from the conus medullaris. Korean J Fam Med 2020;41(1):68-72.

4. Spallone A, Celniku M. Massive Lumbar Disk Herniation Following "Therapeutic" Water Boiling of the Lower Extremities: Case Report and Literature Review. World Neurosurg 2017;97:758-e7.

5. Wang Y, Sun H, Qin S. Clinical efficacy of lumbar interbody fusion using a channel system combined with ozone therapy for the treatment of central-type L3-L4 lumbar disc herniation. Exp Ther Med 2017;13(2):619-23.

6. Notohamiprodjo S, Stahl R, Braunagel M, Kazmierczak PM, Thierfelder KM, Treitl KM, et al. Diagnostic accuracy of contemporary multidetector computed tomography (MDCT) for the detection of lumbar disc herniation. Eur Radiol 2017;27(8):3443-51.

7. Wardlaw D. Sciatica caused by disc herniation: Why is Chymopapain Chemonucleolysis denied to our patients? Int J Spine Surg 2016;10:44.

8. Liu CH, Yeh TC, Kung YY, Tseng HP, Yang CJ, Hong TY, et al. Changes in resting-state functional connectivity in nonacute sciatica with acupuncture modulation: A preliminary study. Brain Behav 2020;10(2):e01494.

9. Boogaard S, Heymans MW, De Vet HC, Peters ML, Loer SA, Zuurmond WW, et al. Predictors of persistent neuropathic pain - a systematic review. Pain Physician 2015;18(5):433-57.

10. Zhang X, Wang Y, Wang Z, Wang C, Ding W, Liu Z. A randomized clinical trial comparing the effectiveness of electroacupuncture versus medium-frequency electrotherapy for discogenic sciatica. Evid Based Complement Alternat Med 2017;2017:9502718.
11. Fernandez M, Ferreira PH. Acupuncture for sciatica and a comparison with Western Medicine (PEDro synthesis). Br J Sports Med 2017;51(6):539-40.

12. Zhai X, Sun C, Rong P, Li S, McCabe MF, Wang X, et al. A correlative relationship between chronic pain and insulin resistance in Zucker fatty rats: role of downregulation of insulin receptors. J Pain 2016;17(4):404-13.

13. Ter Meulen BC, Maas ET, Vyas A, Van Der Vegt M, De Priester K, De Boer MR, et al. Treatment of acute sciatica with transforaminal epidural corticosteroids and local anesthetic: design of a randomized controlled trial. BMC Musculoskelet Disord 2017;18(1):1-8.

14. Xiang A, Xu M, Liang Y, Wei J, Liu S. Immediate relief of herniated lumbar disc-related sciatica by ankle acupuncture: a study protocol for a randomized controlled clinical trial. Medicine 2017;96(51):e9191.

15. Strudwick K, McPhee M, Bell A, Martin-Khan M, Russell T. Best practice management of low back pain in the emergency department (part 1 of the musculoskeletal injuries rapid review series). Emerg Med Australas 2018;30(1):18-35.

16. Oakley PA, Harrison DE. Lumbar extension traction alleviates symptoms and facilitates healing of disc herniation/ sequestration in 6-weeks, following failed treatment from three previous chiropractors: a $\mathrm{CBP}^{\circledR}$ case report with an 8 year follow-up. J Phys Ther Sci 2017;29(11):2051-7.

17. Golbakhsh M, Mottaghi A, Zarei M. Lower thoracic disc herniation mimicking lower lumbar disk disease: A case report. Med J Islam Repub Iran 2017;31:87.

18. Li Z, Yu K, Wang Y, Zhang J. Percutaneous endoscopic transforaminal unilateral decompression for treatment of lumbar spinal stenosis and observational study of non-surgical lower extremity symptoms. Zhongguo Xiu Fu Chong Jian Wai Ke Za Zhi 2019;33(7):831-6.

19. Rodríguez-Vela J, Lobo-Escolar A, Joven E, Muñoz-Marín J, Herrera A, Velilla J. Clinical outcomes of minimally invisual analogue scale ive versus open approach for one-level transforaminal lumbar interbody fusion at the 3-to 4-year follow-up. Eur Spine J 2013;22(12):2857-63.

This is an open access article distributed under the terms of the Creative Commons Attribution-NonCommercial-ShareAlike 3.0 License, which allows others to remix, tweak, and build upon the work non-commercially, as long as the author is credited and the new creations are licensed under the identical terms

This article was originally published in a special issue, "Therapeutic Perspectives in Biomedical Research and Pharmaceutical Sciences and their Nursing Methods"

Indian J Pharm Sci 2021:83(4)Spl issue "5-9" 